\title{
Die europäische Medienlandschaft im Jahr $2000^{1}$
}

\author{
von Albert Scharf
}

Mein Thema verheißt eine Prognose. Dies ist, in der Medienpolitik zumal, ein kühnes Unterfangen. Hektisch verändern sich die Dinge von Woche zu Woche, von Monat zu Monat. Mit Prognosen, so lautet eine englische Weisheit, ist es überhaupt so eine Sache, besonders, wenn sie in die Zukunft gerichtet sind. Andererseits ist diese Zukunft sehr nahe; sie drängt schon heute, denn im Grunde hat sie längst begonnen.

In Deutschland wie in allen europäischen Ländern erleben wir seit einigen Jahren einen dramatischen Auf- und Umbruch der Medienlandschaft. Struktur und Status der Medien hatten sich in dieser kurzen Zeit gründlicher und nachhaltiger verändert als in 60 Jahren davor. Der Anstoß kam von der Technik. Technologie-Sprünge versetzten die Medien gleichsam über Nacht in ein anderes Jahrhundert. Mikroelektronik und Datentechnik verbannten Gutenberg und Marconi ins Museum, schufen neue Arbeitsweisen, neue Berufbilder, neue Mentalitäten. Der Kommunikation wurden in nie gekannter Fülle neue Transportwege erschlossen. Was jahrzehntelang strikter Mangelbewirtschaftung unterlag, öffnete sich zu scheinbarer Beliebigkeit. Wie wenig oder wie viel an Kommunikation möglich war, hing plötzlich nur noch von dem Geld ab, das zu investieren man bereit und in der Lage war.

Breitbandkabel, Nachrichtensatelliten, Mikrochips sind die hauptsächlichen Stichworte dieses faszinierenden Kapitels der Kommunikationsgeschichte, deren Folgen keineswegs nur die Welt des Rundfunks umkrempelten, sondern kaum weniger die Herstellung und Verteilung der klassischen Print-Medien beeinflußten; auch sie sind heute weithin zu elektronischen Medien geworden. Die sprunghafte Erweiterung der Zahl technisch möglicher Transportwege, sprich: Kanäle, die Beschleunigung und Verkürzung der Arbeitsabläufe, die Omnipräsenz weltweit nutzbarer elektronischer Signale haben der Kommunikation nicht nur eine nie gekannte neue Quantität, sondern zugleich eine neue Dimension verschafft.

Und schon schickt sich die Kommunikationstechnologie zu einem weiteren Sprung an, der die Medienlandschaft erneut grundstürzend umgestalten wird. Die "digitale Revolution" steht bevor, die sich bislang

Prof. Dr. Albert Scharf ist Intendant des Bayerischen Rundfunks in München.

1 Vortrag anläßlich des Festaktes „25 Jahre Institut zur Förderung des publizistischen Nachwuchses e. V." am 14.11.1993 in Ludwigshafen. 
erst sporadisch, inselartig, in der Produktion von Tonträgern, ankündigte, die aber die Jahre ab 1995 brachial prägen wird. Digitale Datenkompression vervielfältigt erneut, und zwar schier explosionsartig, die Zahl der Kanäle, mittels derer sich audiovisuelle Inhalte weltweit verbreiten lassen. Aus einem fernsehtauglichen Satellitenkanal lassen sich so, je nach Anspruch an die technische Signalqualität, deren fünf, sechs, sieben, acht entfalten. Multiplizieren Sie diese Zahlen mit den jetzt 30, 40, 50 aus dem Weltall auf uns abgesendeten Satellitenprogrammen, dann haben Sie das technische Szenario, vor das sich die Medien in den nächsten 10 Jahren gestellt sehen. Außerdem: Digitale Telefonsysteme werden mit Datengroßspeichern und Heim-Computern vernetzt und erzeugen zusätzlich eine Komponente des medialen Angebots, die geeignet ist, die Kommunikationsstrukturen und das Kommunikationsverhalten fundamental zu verändern.

Wenn eintritt, was die Propheten dieser weiteren technischen Revolution verheißen, wird der Einzelne zu jeder ihnen beliebenden Zeit jede gewünschte Information, jede Art von Zerstreuung abrufen, jede Kommunikation zu jeder Zeit und zu jedem Zweck herstellen können; das Sesam-öffne-Dich am häuslichen Bildschirm, der über ein Schaltpult mit unzähligen Datenspeichern, Satelliten und Distributionszentralen verbunden ist, der Dialog und prompte, zeitgleiche Lieferung gewährleistet. Und vor dem Bildschirm sitzt staunend, überwältigt, vielleicht aber auch hilflos und verlassen, der total vernetzte einsame Mensch.

Als man hierzulande vor einem Jahrzehnt den Einstieg in neue Kommunikationstechniken auf bewährte deutsche Art mit Pilotprojekten begann, standen ähnliche Visionen schon vor den glänzenden Augen der Zukunftseuphoriker. Ich fragte damals öffentlich, wo denn unter den zahlreichen Ökonomen, Marktforschern und Journalisten der Philosoph sei, der uns sagen kann, ob es gut ist, was wir da treiben. Diese Frage ist mir nicht gut bekommen. Flugs sah ich mich in eine Reihe gestellt mit jenen, die die erste Eisenbahn als Teufelswerk verketzerten. Rasch war ich als Feind des Fortschritts entlarvt und abgestempelt. Und da ich zudem ein Repräsentant der bisherigen Medienstruktur war, war klar, daß solch naives Fragen nur billiger Vorwand für reaktionäres Defensivverhalten sein konnte. Daß einem Menschen, Bürger, Vater, fern aller professionellen Obsession, die gesellschaftlichen Konsequenzen technologischer Innovation, die Wirkungen technisch-industrieller Zwänge auf das Humanum der Gesellschaft ernsthaft bewegen könnten, schien absonderlich und unglaubhaft.

Gewiß, gemessen an der habituellen vordergründigen Unbefangenheit und Gleichgültigkeit gegenüber Technikfolgen, schien meine Frage naiv. Aber die Sorge um den Erhalt humaner Werte, die Suche nach dem, was dem Menschen final wirklich frommt, erscheint oft naiv und unbedarft. Wo leben Sie denn, wird man da gefragt. Und: Wäre es denn nicht auch gut, wenn jeder Mensch seine Kommunikationsbedürf- 
nisse individuell frei gestalten kann, wann und wie es ihm beliebt? Erweitert sich nicht die Sphäre eigenbestimmter Freiheit durch eine beliebige Vielfalt an Quellen der Information, Bildung, Unterhaltung frei von jeder Fremdbestimmung, die der bisherigen Angebotsknappheit unvermeidbar zugrunde lag? Gewiß, würde ich antworten, wenn Gewähr gegeben wäre, daß sich dieser ideale Ansatz in der wirtschaftlich gesteuerten Realität auch verwirklichen ließe. Dafür spricht aber nichts; alle Erfahrung zeigt, daß lediglich gesellschaftlich bewußte Verantwortung durch Marktmechanismen ersetzt wird.

Die neue Vielfalt zersetzt sich in der Beliebigkeit von Angeboten, soweit sie sich marktwirtschaftlich rechnen; andere gibt es nicht. Und selbst wenn gelänge, was Euphorie uns verheißt: Was wäre dies für ein Mensch, der da vor seinem Bildschirm sitzt und so die Welt erfährt und bewältigt? Ist diese absolute Zuspitzung technischer Möglichkeiten dem "animal sociale" noch wesensgemäß? Wird er sich dieser Kommunikation unterwerfen? Wird er sich nicht womöglich entziehen, aus der Isolation auf seiner Schaltinsel lösen und sich so dem Markt verweigern? Übrig bliebe, wie gewohnt, das Massenattraktive: die Masse bestimmt, was den einzelnen angeboten wird.

Die Auswirkungen solch größerer Pluralität und Kommunikationsvielfalt erleben wir zur Zeit in vergleichsweise kleinem Maßstab und erfahren dabei bereits eindrücklich die - wie C. F. von Weizsäcker es abgeklärt nennt - "Ambivalenz des Fortschritts". Der Januskopf technischer Innovation ist uns täglich vor Augen. Die immerzu steigende Vervielfachung technischer Möglichkeiten der Telekommunikation, die das rastlose Ingenium der Ingenieure uns beschert, haben schon jetzt aus einer öffentlichen Dienstleistung einen Markt entstehen lassen, auf dem nicht mehr ein soziales, gesellschaftliches Bedürfnis das Angebot bestimmt, sondern das technisch nur je Denkbare die Nachfrage einfordert; bietet diese Nachfrage keinen ausreichenden Ertrag, keine Profilperspektiven, entfällt das Angebot, auch wenn ein Bedürfnis bestehen sollte. Liberalisierung nennt man dies. Das Schlagwort will Freiheit und Freizügigkeit insinuieren.

In Wirklichkeit gerinnt der so verführend in Aussicht gestellte Prozeß einer Liberalisierung und Individualisierung der Kommunikation zu einer Diktatur marktwirtschaftlicher Zwangsläufigkeiten: Sinnvoll ist nicht mehr allein, was der Gesellschaft dient, sondern, was ein Geschäft verspricht, Gewinn verheißt, profitabel ist. Wettbewerb heißt die Devise, Wettbewerb als nachfrageorientiertes Regulativ des Angebots. Aufklärerischer Idealismus erhoffte sich vom freien Wettbewerb die freie Selektion des Besseren. Tatsächlich verhilft der Wettbewerb zunächst einmal dem wirtschaftlich Stärkeren zum Erfolg, zumal die Feststellung von Qualität, des Besseren, Bewertungskriterien voraussetzt, auf die man sich offenbar so leicht nicht verständigen kann, wenn es um Geist und Inhalte geht. Entsprechend wertfrei, um nicht zu sagen "wertelos", definiert sich auch Wettbewerb. 
Wettbewerb sei, so sagt uns schon das Grimm'sche Wörterbuch, „das Verhältnis solcher Personen zueinander, die mit Aufwand irgendwelcher Mittel einen Zweck erstreben, den nicht alle erreichen können". Daraus ließe sich - auch ohne alle naheliegende Polemik - die heutige Problematik und die ganze Spannbreite meines Themas kritisch entwickeln. Um welchen Zweck geht es? Rechtfertigt der Zweck alle Mittel? Bestimmt nicht am Ende der Wettbewerb den Zweck?

Diese Feststellung wird die Medienlandschaft Europas in der nahen Zukunft entscheidend beeinflussen. Überall hat sich im Bereich des Rundfunks ein aggressives Nebeneinander kommerzieller und öffentlicher Programmveranstalter entwickelt, die sich - auch wenn und wo dies nicht politisch gewollt sein sollte - einen Verdrängungswettbewerb zu liefern haben. Kommerzielle, kapitalistische Machtzusammenballungen konzentrieren auch bei Zeitungen, Zeitschriften und Buchverlagen den entscheidenden Wettbewerb um Marktanteile und Werbeerlöse auf letztlich wenige Agglomerationen, die mit hohem Kapitaleinsatz Exklusivität horten und in engen Ketten der - wie Herr Lahnstein dies geziemend vornehm formuliert - "Ressourcenoptimierung" den Wettbewerb keineswegs pflegen, sondern beschränken: Senderfamilie, Verlagspalette, Medienverbund nennt man das, was in Wirklichkeit knallharte Monopolpolitik ist. Aber unter der freiheitlich verbrämten Wertmarke "freie Marktwirtschaft" läßt sich trefflich private Interessenpolitik betreiben.

Auch als soziale Marktwirtschaft steht sie als Prinzip einer Entwicklung nicht entgegen, die zuweilen verteufelt an das erinnert, was man einmal Manchester-Liberalismus genannt hat: Über Wert und Unwert entscheidet der wirtschaftliche Erfolg. Daß dieses Kriterium für kulturelle Phänomene denkbar ungeeignet ist, wird geflissentlich verdrängt. Dies ist das Verhängnis, das der technisch so freizügig gefüllten Büchse der Pandora entfleucht in dieser europäischen Medienentwicklung heute und morgen. Das in Festreden so gerne bekundete Bekenntnis zur Bedeutung der Medien als Bestandteil europäischer Kultur und Identität verflüchtigt sich in der Realität wirtschaftspolitischer Prioritäten und offenbar zwanghaft folgender medienpolitischer Praxis.

Ich will gar nicht bestreiten, daß die Öffnung des Rundfunkmarktes begleitet war von der allseitigen Einsicht in die Notwendigkeit eines Rahmens gesellschaftlicher und kultureller Verantwortung. Die Mahnung des Bundesverfassungsgerichts, daß der Rundfunk ein zu wichtiges, gesellschaftswichtiges Gut sei, als daß er einfach dem freien Markt überlassen werden dürfe, hat in Deutschland immerhin zu einem staatsvertraglichen Ordnungsrahmen geführt, der besorgte Geister beruhigen konnte. Doch die Realität hat diese Intentionen rasch überholt und demnächst wird der Overspill hunderter von Satellitenkanälen nationale Reglementierungen zum Mahnfaktor werden lassen. Übrig bleibt vielleicht der Effekt der Domestizierung nationaler Veranstalter. 
Aber diese werden sich mit dem Verweis auf die Libertínage anderswo entziehen. So wird, ich fürchte, wie bisher schon, um nur ja den selbstbestätigenden wirtschaftlichen Erfolg nicht zu behindern, „Laissez faire - laissez passer" die Parole progressiver Medienpolitik bleiben. Als sich schon im ersten Durchgang einstellte, was nun allenthalben zu hören und zu sehen ist, brach zuweilen betretene Überraschung aus: wer hätte das gedacht! So haben wir uns dies nicht vorgestellt! - Wie demu sonst, möchte ich fragen.

Mehr Vielfalt hatte man sich versprochen, mehr innovative Kreativität, vielleicht auch freundlichere Information, weniger Kritik und Widerspruch, mehr Positives. Statt dessen entsteht mehr Einförmigkeit, um nicht zu sagen Einfalt. Eine neue Art Journalismus drängt sich auf, mal flapsig, mal aggressiv, massierte Trivialität macht sich breit. Gewalt und Sex dienen als publikumsträchtige Reizmittel. Ich bestreite nicht, daß es einige Beispiele gibt, die man als Bereicherung des Rundfunkangebots empfinden kann - aber um welchen Preis! Und als Ausnahmen bestätigen sie nur die unsägliche Regel. Kein Land in Europa, das nicht das gleiche deprimierende Bild bietet. Das alte Monopol gesellschaftlich verantworteten öffentlichen Rundfunks wurde zerschlagen, um neuen, wirtschaftlich ungleich potenteren Mono- oder Oligopolen Platz zu machen.

Dabei war natürlich vorhersehbar, was sich bei Einführung des kommerziellen Rundfunks einstellen würde; der Erfahrungen gab es weltweit genug. Das Risiko, zumindest das Risiko, wenn schon nicht die Gewißheit, eines nach unten abgleitenden Rundfunkstandards, das Risiko von Auswüchsen eines so freien Marktes, ist dem freien Wettbewerb immanent und dem geschichtlich erfahrenen Nationalökonomen bekannt.

Im "Conversations-Lexikon" von Brockhaus aus dem Jahre 1884 steht unter dem Stichwort „Konkurrenz":

„Die freie Konkurrenz entfesselt mit guten Kräften zugleich die schlechten, und gelegentlich hat es sich gezeigt, daß die letzteren überwiegen. Man hat als freie Konkurrenz angesehen, im Interesse des Gewinns alles das thun zu dürfen, was durch das Strafrecht nicht ausdrücklich verboten ist. Die Konkurrenz führt vielfach zur inneren Verschlechterung der Produkte bei Wahrung des äußeren Scheins, also zu einer unsoliden Produktion, auf welche das Schlagwort, billig und schlecht ${ }^{\prime}$ paßt.

Obenein zeigt sich neuerdings, daß aus der freien Konkurrenz mehr und mehr einzelne Übergroße hervorwachsen, mit welchen es die kleinen Konkurrenten auf die Dauer nicht aufnehmen können; ferner nimmt man wahr, daß in zahlreichen Fällen die geträumte Konkurrenz überhaupt nicht stattfindet, sondern Verabredung, Koalition, Kombination (s. Monopol)." 
Soweit das Zitat, das, wohlgemerkt, aus dem Jahr 1884 stammt. Ist es nicht eine hellsichtige Beschreibung all der Merkmale unserer heutigen Medienszene in Europa?

Damit man mich nicht mißverstehe: Ich bin nicht gegen Marktwirtschaft und nicht gegen Wettbewerb. Auch im zitierten „Brockhaus" endet der Artikel mit dem Satz:

„Wenn aber auch solche Auswüchse und Mißstände nach Möglichkeit zu bekämpfen sind, so bleibt doch die Konkurrenz eine in der bestehenden wirtschaftlichen Ordnung unentbehrliche Treibkraft."

Wettbewerb wird auch das maßgebliche Schlagwort der Medienpolitik in den vor uns liegenden Jahren sein. Die Realität der Medien wird letztlich wirtschaftspolitisch definiert. Dies ist entlarvend und entwaffnend zugleich. Jedenfalls ist dies die leichteste, bequemste Art, jede gesellschaftspolitische Bedenklichkeit rigoros beiseite $\mathrm{zu}$ schieben: Arbeitsplatzsicherung, Exportrate, Investitionskraft, Geldwertstabilität, Baisisindustrien, Standort Europa, Deutschland, Berlin, Köln, München ..., dies sind Argumente, die jeden Gesellschafts- und Kulturphilosophen herrisch des Feldes realpolitischer Auseinandersetzung verweisen.

Dennoch: die Reaktion auf dieses technisch-wirtschaftlich-politische Szenario kann, darf und muß nicht die resignativ-nostalgische Einsicht sein, daß dies alles zwar von Übel, aber halt unabänderlich sei. Wir begehren nur, mit Grillparzers Worten, nicht schuld daran zu sein. Es genügt aber nicht, Schlimmes zu diagnostizieren und sich dann betrübt abzuwenden. Realitäten muß man sich stellen, um zumindest den Versuch zu wagen, sie zum Besseren zu steuern.

Den technischen Fortschritt, oder was man dafür hält, wird niemand aufhalten. Gewiß müßte der Mensch nicht alles machen, was er kann. Aber er tut es eben doch und sei es auch nur, um sich zu beschäftigen, sich Arbeit $\mathrm{zu}$ verschaffen, un materielle Werte zu schöpfen, von denen er lebt und denen er gerne verfallen ist.

Also kann es nur darum gehen, diese Technik auch in den Dienst des Nützlichen, Wichtigen, des Allgemeinen, nicht nur je privaten Wohles zu stellen. Man muß Instrumente erhalten oder schaffen, die uns in die Lage versetzen, in die Springflut unüberblickbarer medialer Beliebigkeit auch Inhalte von Gewicht einzubringen, Werte womöglich, Werte, von denen Profil und Rang der Gesellschaft abhängen. In dem Sortiment unterschiedlicher Dienste, Sparten und Programme müssen wir Sorge tragen, daß es auch Kommunikationsangebote gibt, die Wegweisung, Standorte, Orientierung bieten und der "verzweifelten Langeweile der Seele" entgegenwirken, die schon Nietzsche als Folge der seelenlosen Tätigkeit an der Maschine vorhergesagt hat. Insoweit ist das Maschinenzeitalter noch nicht abgeschlossen. Es hat nur einen anderen, diskreteren, individualisierten Charakter angenommen. In der 
Tat suchen die Menschen, umstellt von wesenlosen Maschinen, Computern und Datenbanken, eingesponnen in einem Netz von Bits und Bytes, vermehrt und geradezu süchtig nach objektiver, vorurteilsfreier Information; sie suchen auch Besinnung, Muße, Lebenserfüllung, sie suchen das Schöne, das Bewährte und Bewahrenswerte. Sie wollen nicht nur Objekte rationaler Kalkulation und Spekulation sein; sie wollen als Menschen ernstgenommen werden.

In dem Maße, wie Information und Kommunikation jeder Art sich mit Hilfe einer leviathanischen Technik zur erdrückenden Masse ballt, wächst die Verpflichtung, die Inhalte dieser Kommunikation zu ordnen, nicht nur sichtbar, sondern auch transparent und durchsichtig zu machen. Dazu braucht man mehr denn je Kommunikatoren und Informatoren, die sich ihrer Aufgabe redlich, unvoreingenommen, aufrichtig in bescheidener Zurückhaltung widmen.

Wir haben uns angewölnt, davon $\mathrm{zu}$ reden, daß wir, die Menschen am Ende des 20. Jahrhunderts, in einer "Informationsgesellschaft" leben. Wir sagen das meist sogar, wie es scheint, mit einem gewissen Stolz: wie herrlich weit wir es damit gebracht haben, unendlich viel mehr zu erfahren als unsere Vorfahren, ja womöglich alles zu erfahren und alles zu wissen - eine Welt voll aller Erfahrung und aller Erlebnisse, offen, transparent, allgemein zugänglich. Es klingt ja auch viel besser als "technisierte Gesellschaft “, „Industriegesellschaft ${ }^{\text {" }}$ oder gar "Massengesellschaft"; nein, fortschrittlich, optimistisch nennen wir uns jetzt eine "Informationsgesellschaft". Das Traumbild jedes aufklärerischen Geistes und doch - welch ein Irrtum! Wir sind uns natürlich dieses Irrtums durchaus bewußt. Wissend oder doch zumindest ahnend, sind wir aufgeklärt und informiert genug, um uns eher unbehaglich zu fühlen angesichts der schier unermeßlichen Fülle von Daten, Fakten, Meinungen, Hintergründen und Bezügen, von der wir tagtäglich überschwemmt werden.

Die "mirabilia technicae inventa", wie die Pastoralinstruktion "Communio et progressio" die Kommunikationstechnik liebenswürdig staunend nennt, diese wundersamen Erfindungen der Technik haben uns eine Überfülle an Informationen, Bildern und Eindrücken geliefert, die uns heute an jedem Zeitungsstand entgegentritt, die uns aus jedem Radio- oder Fernsehgerät zu jeder Tages- oder Nachtzeit überfällt. Wer zählt noch die Programme und Kanäle, die uns informieren und, vor allem, unterhalten wollen - aber auch das, was sich als Unterhaltung gebärdet, ist Information, menschliche Mitteilung, die unseren Geist, unser Verhalten, unsere personale Entwicklung beeinflußt. Die ganze Welt ist bei uns daheim. Wir sind scheinbar überall dabei, live und unmittelbar. Die Welt ist, wie Marshall McLuhan richtig vorausgesagt hat, vor allem durch die elektronischen Medien, zum "global village" geschrumpft. Ein Netzwerk von Informationen umfängt uns, aus dem es kaum noch ein Entrinnen gibt. 
Neil Postman, der scharfzüngige Kulturkritiker, der uns Mitte der 80er Jahr schon einmal gewarnt hatte, daß unsere freie Urteilsbildung im Showgeschäft unterginge, weil wir uns zu Tode amüsieren, hat kürzlich ein neues Buch vorgelegt, in dem er die Entmündigung der Gesellschaft durch das "Technopol“ beschreibt. Das Technopol ist für Postman "die totalitär gewordene Technokratie", die "das, was wir unter Religion, Kunst, Familie, Politik, Geschichte, Wahrheit, Privatsphäre, Intelligenz verstehen, neu definiert, dergestalt, daß die Definitionen schließlich den Anforderungen des Technopols genügen“. Mit anderen Worten: die total und totalitär gewordene Technokratie zwingt uns in ein anderes Verständnis der Welt und unser selbst; sie verändert gesellschaftliches Bewußtsein, sie führt zur Entmündigung des Menschen, weil sie alle Formen des kulturellen Lebens der Vorherrschaft von Technik und Technologie unterwirft.

Fortschritt der Menschheit - Fortschritt zum Besseren? Ist der besser informierte Mensch auch ein besserer Mensch, ist die besser informierte Welt eine bessere Welt? Sind wir überhaupt besser informiert oder nur umfänglicher? Gerät uns das Fortschreiten der Technik zum Segen oder Fluch, zum Heil oder Unheil?

Seien Sie unbesorgt: ich kann und will mich nicht weiter in diese fundamentalen gesellschaftsphilosophischen Fragen verlieren. Andere, Gescheitere haben dies schon reichlich und kontrovers genug getan. Meinungen und Prognosen aller Art sind uns in großer Zahl angeboten: pessimistische, optimistische, realistische, idealistische. Auch darüber sind wir informiert.

Allein, dieses Beispiel führt vor Augen, was Unbehagen schafft: eine schier unübersehbare Vielfalt von Informationen, von Daten, Berichten, Meinungen liegt uns vor. Haben wir aber in all diesen Dingen auch eine eigene, selbst geformte Meinung? Bietet uns all diese Informationsfülle auch genügend Orientierung zur eigenen Meinungsbildung, zur Entscheidung über das, was wir je für uns selbst entscheiden müssen, was wir für richtig halten wollen - oder führt uns die Vielfalt einer allen zugänglichen Information in einen Dschungel, in dem uns kein eigener Weg mehr möglich ist, in dem wir nur noch den vorgegebenen, ausgetretenen Trampelpfaden der Trends, der Durchschnitts- und Mehrheitsmeinungen folgen können, wie manipulativ diese Wege auch immer vorgestaltet wurden? Verlieren wir also im für uns undurchdringlichen Dschungel einer technologisch bedingten allumfassenen Informationsgesellschaft die geistige Unabhängigkeit, die den Menschen in seiner personalen Würde ausmacht? Was wissen wir eigentlich bei oder trotz all dieser überwältigenden Menge an Information?

Zweifel, Skepsis sind jedenfalls angebracht. Der Konstanzer Philosoph Jürgen Mittelstrass schreibt in der "Neuen Zürcher Zeitung“ vom 23.01.1992, wir Menschen unserer Zeit seien Informationsriesen 
und Wissenszwerge zugleich: „Der Informationswelt entspricht, strukturell und auf den einzelnen Konsumenten der Information bezogen, eine Meinungswelt, keine Wissenswelt; der Informierte selbst weiß nicht, ob er in einer Wissenswelt oder in einer Meinungswelt lebt. Das wiederum eröffnet eine unerwartete Gefahr einer neuen Dummheit. Wer sich auf Informationen verläßt, die er selbst (als Wissender) nicht zu prüfen vermag, könnte der Dumme sein.“

Wenn das so ist, und ich fürchte, weithin wird es so sein, dann müssen wir uns fragen, was können, was müssen wir tun, um am Ende eben nicht die Dummen zu sein? "Wir" heißt, jeder von uns; vor allem aber wir, die wir Verantwortung tragen für das, was veröffentlicht wird, als offenes, freibleibendes Angebot zwar, aber doch aufdringlich und unentrinnbar.

Die Medien, die dem Menschen diese Kommunikationsüberfülle bescheren, müssen Orientierungshilfe leisten. Sie dürfen nicht nur mitteilen, was ist und wer was gesagt hat, sondern müssen ebenso versuchen $\mathrm{zu}$ vermitteln, warum etwas ist oder gesagt wurde. Sie müssen Zusämmenhänge, Hintergründe, Bezüge auftun, Zusammenhänge herstellen, Fragwürdiges hinterfragen und so - im doppelten Sinn der Worte - aufklären, Einsicht verschaffen.

Unverzichtbare Voraussetzung für diesen Dienst der Medien an der Gesellschaft sind die Grundtugenden eines redlichen Journalismus: Unvoreingenommenheit und Wahrhaftigkeit in der Berichterstattung, Sachkunde und Überblick in der Analyse, Fairness im Kommentar, angemessene Vielfalt bei der Vermittlung von Meinungen und Standpunkten, Sorgfalt der Sprache, und zwar im Begrifflich-Inhaltlichen wie in der simplen Grammatik. Offen und unabhängig mußt der Informationsvermittler sein, frei und mutig, aber auch bescheiden und selbstkritisch, selbstbewußt, aber nicht zu selbstsicher, schon gar nicht hoch- und übermütig arrogant.

Wie die Gesellschaft die ihr zugängliche Information verarbeitet und verkraftet, wie sie schließlich öffentliche Meinung bildet, wie wir es also mit der sozialen Kommunikation halten, ist eine Schicksalsfrage der modernen Massengesellschaft. Diese Masse in all ihrer Pluralität und Polarisierung kann Gesellschaft, kann Gemeinschaft, Geschichts- und Schicksalsgemeinschaft nur werden und bleiben, wenn sie durch sachgerechte, tolerante und faire Kommunikation dazu befähigt wird, wenn sie eine Information und Orientierung erhält, die - um mit Otto B. Roegele zu sprechen - dem Leser, "dem Hörer und Zuschauer nicht nur die Länge, Breite und Fülle der Nachrichten vermittelt, sondern auch die Abszisse und Ordinate eines Bezugssystems, in das sich die einzelnen Nachrichten eintragen lassen, damit sie verstanden, $d . h$. in ihrer Bedeutung und in ihrem Zusammenhang erkannt werden können". 
Die Bedeutung dieser gesellschaftlichen Funktion der Medien und Mediatoren kann gar nicht hoch genug eingeschätzt werden. Anfang der 60er Jahre sagte Karl Jaspers in einer Sendereihe des Bayerischen Rundfunks über die damals schon genauso besorgt gestellt Frage „Werden wir richtig informiert?": „Was für Journalisten ein Volk hervorbringt, das ist heute ein wesentliches Moment seines Schicksals."

SUMMARY: The European landscape of media in the year 2000

In a retrospective on the introduction of private television in Germany ten years ago, the superintendent of the 'Bayerischer Rundfunk' questioned the profile and perspectives of the future development of the media. The author complains about the underdeveloped state of ethical and philosophical evaluation of the massive technological progress of recent years. In his point of view the future media user is going to be a socially isolated man in front of a multifunctional television screen.

As a result of the "liberalisation" of social communication, he asks for the limits of a general liberty of competition and free profit orientation. The social task of broadcasting is a good too prescious to distribute it only among financial and economical interests. Broadcasting has to create a public opinion, which by means of appropriate, tolerant and fair communication contributes to inform people and give them orientation for their lives.

\section{RÉSUMÉ: Le paysage audio-visuel européen en l'an 2000}

En faisant une rétrospective de l'introduction du système radiophonique binaire en Allemagne il y a 10 ans, l'intendant de la radio bavoroise se demande quels sont le profil et les perspectives de l'évolution audio-visuelle dans un avenir proche. En prenant en considération les progrès technologiques énormes auxquels on assiste à notre époque, l'auteur se plaint qu'on ne se pos pas assez la question de savoir quelle est la valeur éthique et philosophique que l'on doit attribuer à cette évolution. Il craint que l'homme devant le télécran soit complètement "câblé" et socialement isolé.

A la suite de la "libéralisation" de la communication sociale, il se demande où sont les limites $d^{\prime}$ une liberté générale de la concurrence et $d^{\prime} u n e$ orientation vers un profit démesuré. La tâche sociale de la radio est un bien trop précieux pour qu'il soit livré aux seuls intérêts économiques et politicofinanciers. La radio doit former une opinion publique qui contribue, grâce à une communication objective, tolérante et loyale, à donner aux hommes à las fois information et orientation.

RESUMEN: Los medios de comunicación europeos en el año 2000

Mirando en retrospectiva la introducción del sistema dual de radioemisión hace 10 años, el intendente de la ,Bayerischer Rundfunk' se pregunta por el perfil y las perspectivas del desarrollo de los medios en próximo futuro. El autor deplora que, considerando el masivo desarrollo tecnológico del presente, la problemática acerca de una valoración ética y filosófica de este desarrollo no despierte un interés mayor. El teme que el ser humano termine como un ser mefido en múltiples redes, socialmente aislado y frente a un televisor.

Como consecuencia de la "liberalización" de la comunicación social, el 
plantea la pregunta acerca de las fronteras de una libertad de competencia general y de una deshinibida búsqueda de la ganancia económica. La tarea social de las emisoras es un bien muy valioso como para dejarlo a disposición de los intereses político-económicos o político-financieros. Las emisoras deben formar la opinión pública a través de una información objetiva, tolerante y adecuada, haciendo su aporte de esta manera a la tarea de dar a la gente información y orientación. 